\title{
The role of vaccines in preventing bacterial antimicrobial resistance
}

\author{
Kathrin U Jansen, Charles Knirsch \& Annaliesa S Anderson
}

\begin{abstract}
Antimicrobial resistance (AMR) and the associated morbidity and mortality due to bacterial pathogens have been increasing globally to alarming levels. The World Health Organization (WHO) has called for global action on AMR, supported worldwide by governments, health ministries and health agencies. Many potential solutions to stem AMR are being discussed and implemented. These include increases in antimicrobial stewardship, investment in research and development to design new classes of antibiotics, and reduction of antibiotic use in rearing of livestock. However, vaccines as tools to reduce AMR have historically been under-recognized in these discussions, even though their effectiveness in reducing disease and AMR is well documented. This review article seeks to highlight the value of vaccines as an additional modality to combat AMR globally, using select examples. It also will provide perspectives on how vaccines could be more effectively used in this effort.
\end{abstract}

In recent history, an unacceptable increase in AMR has arisen, with evidence of bacteria becoming resistant to not only one, but multiple classes of antibiotics ${ }^{1}$. Once antibiotic-resistant strains emerge, they can spread rapidly around the globe and acquire resistance to additional drug classes (Table 1). Multidrug-resistant (MDR) strains are associated with an increase in morbidity and mortality ${ }^{2}$.

In most cases, antimicrobial resistance (Box 1) is the result of the select response mechanisms of pathogens to the presence of antimicrobial drugs, the use of which is globally widespread and often deviates from guidance provided in the label. The emergence of bacterial antibiotic resistance generally occurs soon after the clinical introduction of new antibiotics. As such, resistance has been reported to be highest in regions that have high per capita use of these therapeutic drugs $^{3}$. However, the impact of resistant organisms is evident even in countries in which per capita drug use is low. This resistance has dramatic consequences, such as clonal outbreaks of resistant organisms impacting patient outcomes ${ }^{4}$.

It is estimated that by 2050, 10 million lives a year may be lost to AMR, exceeding the 8.2 million lives a year currently lost to cancer $^{5}$. To put this number in perspective, currently, at least 700,000 people die of resistant infections every year globally, more than the combined number of deaths caused by tetanus, cholera and measles. This burden is also not far behind the mortality due to common afflictions such as diarrheal diseases or diabetes. There are a number of drivers responsible for this situation, such as increases in global antibiotic use ${ }^{6}$; lack of universally applied best practices in antibi-

Pfizer Vaccine Research and Development, Pfizer, Inc., Pearl River, New York, USA. Correspondence should be addressed to K.U.J. (kathrin.jansen@pfizer. com).

Received 19 June 2017; accepted 4 December 2017; published online 9 January 2018; doi:10.1038/nm.4465 otic stewardship and education ${ }^{6,7}$; inappropriate use of antibiotics in medical practice, such as underdosing and prescriptions to treat minor bacterial infections or viral infections ${ }^{8}$; and in addition the widespread and uncontrolled use in animals to increase meat production ${ }^{9}$.

It has been estimated that, if the AMR trend continues, the cumulative loss to world economies might be as high as $\$ 100$ trillion by 2050 (ref. 5). While renewed efforts in research and development to discover the next wave of antibacterial drugs are vital in addition to globally practiced stringent antibiotic stewardship, vaccines are starting to be recognized as essential and highly effective tools to mitigate $\mathrm{AMR}^{10}$. The prophylactic use of bacterial vaccines prevents bacterial infections, thus reducing the need for antibiotic prescriptions and minimizing the selective drug pressure that can result in resistant strains (Fig. 1). In addition, positive effects of vaccines on AMR have been observed with viral vaccines, such as those to prevent influenza ${ }^{11}$. Such vaccination can result in a reduction in inappropriate antibiotic prescriptions for a viral disease and also lower the frequency of bacterial superinfections that would require antibacterial therapy.

In the following sections, we will describe the epidemiology of bacterial AMR and discuss the roles vaccines can play in addressing AMR, giving select examples of vaccines already licensed to prevent infections with Haemophilus influenzae type b (Hib), Streptococcus pneumoniae and influenza. Furthermore, we will provide to the reader a brief review of vaccines in later stages of clinical development that have the potential to further reduce AMR, if successfully developed, such as vaccines to prevent infection with respiratory syncytial virus (RSV), Clostridium difficile, Staphylococcus aureus and group B Streptococcus. Finally, we will touch on the need to develop vaccines to prevent infections with Gram-negative bacteria and Mycobacterium tuberculosis and will describe the barriers as well as prospects to developing such vaccines. 
Table 1 Global antibiotic resistance levels associated with major bacterial pathogens

\begin{tabular}{|c|c|c|c|c|c|}
\hline \multirow[b]{2}{*}{ Pathogen } & \multicolumn{5}{|c|}{ Resistance rate } \\
\hline & United States & S. Africa & UK & India & Australia \\
\hline S. pneumoniae & $17-34$ & ND & $7-8$ & ND & ND \\
\hline S. aureus & $0-45$ & $0-29$ & $0-11$ & $2-94$ & $0-18$ \\
\hline E. coli & $1-55$ & $0-84$ & $0-66$ & 11-92 & $0-55$ \\
\hline Enterobacter spp. & $5-88$ & $3-100$ & ND & ND & $3-30$ \\
\hline K. pneumoniae & $8-22$ & $2-68$ & $0-14$ & $2-80$ & $0-9$ \\
\hline$P$. aeruginosa & $5-26$ & $1-35$ & $3-14$ & $0-69$ & ND \\
\hline A. baumannii & $6-49$ & $2-41$ & $2-9$ & $3-90$ & ND \\
\hline M. tuberculosis & $0-2.9$ & $3-5.9$ & $0-2.9$ & ND & $0-2.9$ \\
\hline N. gonorrhoeae & $0.1-3$ & $0.1-70$ & $0-70$ & $0.1-70$ & $0.1-70$ \\
\hline
\end{tabular}

Antibiotic resistance data are presented from 2000-2014 and listed as the percentage of isolates tested that were resistant to each antibiotic class used for each pathogen. These antibiotics are pathogen specific. The table depicts the range of antibiotic resistance for each pathogen via available drugs and does not take into account the proportion of strains that are resistant to more than one antibiotic class. Data for all pathogens except $M$. tuberculosis and $N$. gonorrhea were obtained from the Center for Disease Dynamics, Economics and Policy (https://resistancemap.cddep.org/). Tuberculosis data were obtained from ref. 85. N. gonorrhea data were obtained from the WHO Gonococcal Antimicrobial Surveillance Programme (GASP), which covers strains analyzed between 2011 and 2014 (http://www.who.int/ reproductivehealth/topics/rtis/gonococcal_resistance/en/). ND, no data provided.

\section{Epidemiology of bacterial antimicrobial resistance}

The introduction of penicillin in the 1940s allowed for the treatment of often fatal infections such as sepsis, cellulitis, pneumonia and endocarditis caused by organisms such as streptococcal species or $S$. aureus. S. pneumoniae was first noted to develop resistance to penicillin, by producing $\beta$-lactamases that render penicillin inactive, in South Africa in 1977 (Box 1). The resistance in this organism subsequently spread worldwide as the protein targets of the antibiotic continually mutated ${ }^{12}$. Pneumococci with increasing resistance to the third-generation cephalosporins commonly used to treat meningitis were subsequently reported to the US Centers for Disease Control and Prevention (CDC) in the $1990 \mathrm{~s}^{13}$.

S. aureus is another example of a pathogen that was noted to produce $\beta$-lactamases, resulting in the development of resistance to new generations and classes of antibiotics, including methicillin ${ }^{12}$. Treatment of methicillin-resistant $S$. aureus (MRSA) with vancomycin and increased empirical use of this antibiotic for suspected meningitis cases provided the antibiotic pressure associated with the emergence of vancomycin-resistant enterococci and vancomycin-intermediate-resistant staphylococci ${ }^{14}$. C. difficile has developed resistance to the first-line recommended antibacterial agent metronidazole ${ }^{15}$, yet it remains susceptible to vancomycin and fidaxomicin. Historically, routinely prescribed quinolone antibiotics were associated with the rapid global spread of a quinolone-resistant epidemic strain of $C$. difficile, resulting in the high morbidity and mortality associated with this disease ${ }^{16}$.

Among the WHO list of priority pathogens (http://www.who.int/ mediacentre/news/releases/2017/bacteria-antibiotics-needed/en/), defined as the antibiotic-resistant pathogens posing the greatest threat to human health and for which the development of new antibiotics is urgently needed, are the Gram-negative Enterobacteriaceae, organisms such as Escherichia coli, Klebsiella pneumoniae, Enterobacter spp., Serratia spp., Proteus spp., Providencia spp. and Morganella spp. Their global levels of resistance to different classes of antibiotics are illustrated in Table 1. Enterobacteriaceae cause a wide range of infections, including pneumonia, urinary tract infection, bacteremia and surgical site infection ${ }^{17}$. Even the clinically ubiquitous Gram-negative bacterium E. coli, an organism associated with routine outpatient urinary tract infections and bacteremia, has developed resistance to multiple classes

\section{Box 1 Molecular mechanisms of antibiotic resistance}

Antibiotics either kill bacteria (bactericidal) or stop them from growing (bacteriostatic). Antibiotic mechanisms of action are based on (i) prevention of DNA or RNA synthesis, (ii) prevention of folate synthesis, thereby blocking nucleic acid synthesis, (iii) destruction of the cell wall/membrane and (iv) prevention of protein synthesis by interfering with ribosome function.

Antibiotic resistance mechanisms elaborated by bacteria counteract the effectiveness of antibiotics. Resistance mechanisms can be acquired by horizontal transfer of plasmids or other genetic elements from bacteria that are colocalized with the pathogen. Alternatively, resistance can occur through vertical transmission by chromosomal mutations.

Resistance mechanisms may include expression of enzymes such as the $\beta$-lactamases, which inactivate $\beta$-lactams, or removal of the antibiotic by efflux pumps. Alternatively, the target of the antibiotic may be modified so that the antibiotic can no longer bind or interact with the target. Bacteria can also have bypass mechanisms that circumvent antibiotic toxicity. For example, they can modify the cell surface to prevent antibiotic entry. In addition, bacteria can directly modify antibiotics to prevent target engagement.

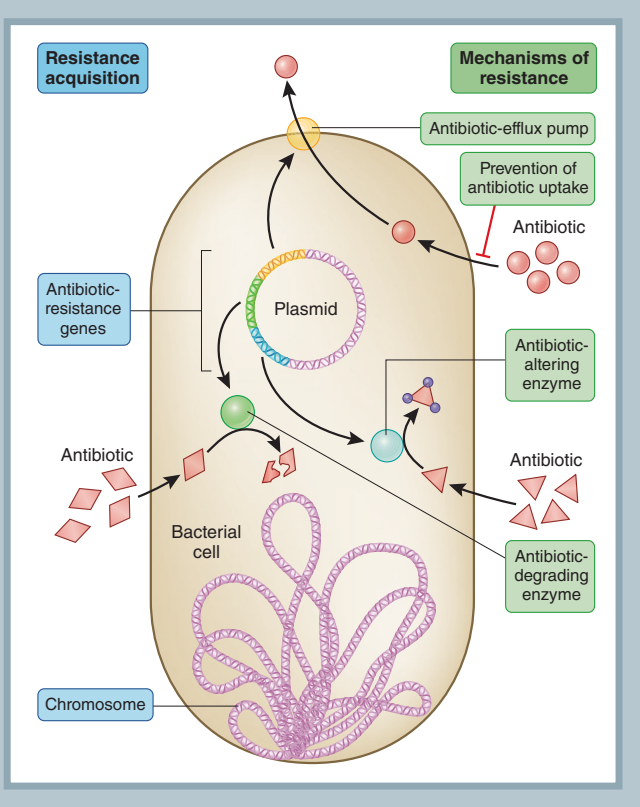




\section{REVIEW}

a

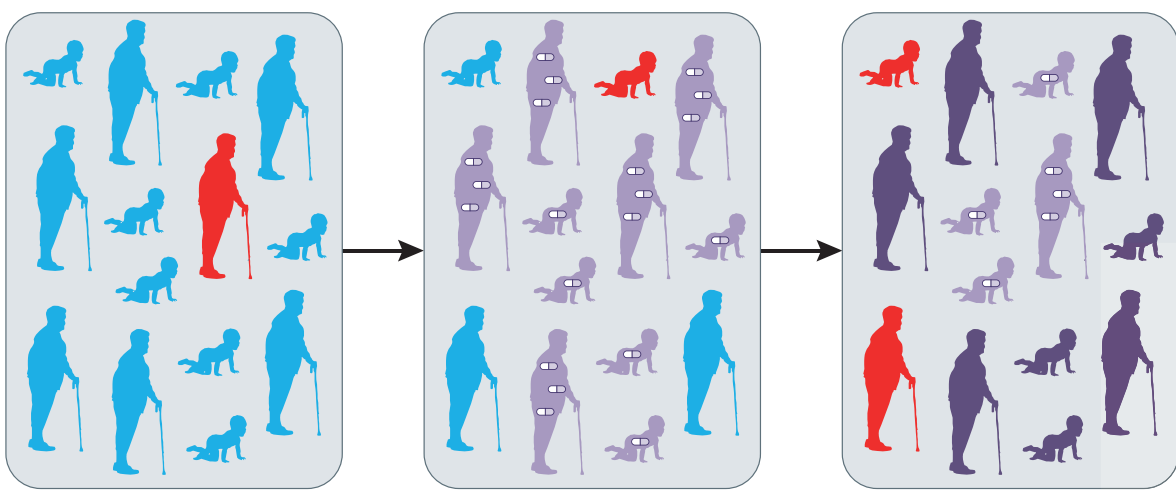

\begin{tabular}{|l|}
\hline Infected \\
Immunized \\
Susceptible \\
$\square$ Infected and taking abx \\
Abx resistant
\end{tabular}

b

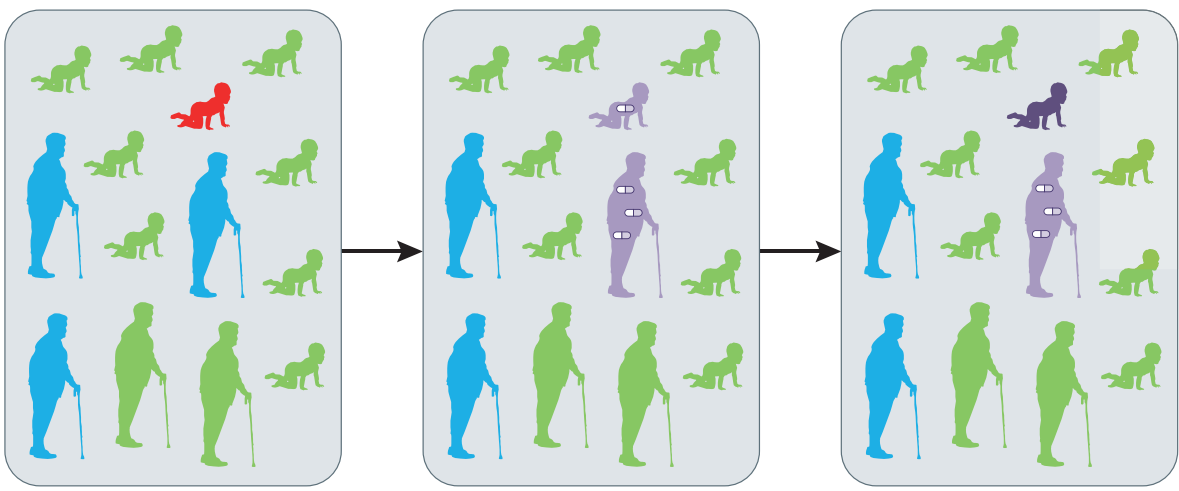

Abx resistant population is not immunized against a bacterial pathogen, most members of the population are susceptible to the pathogen. A susceptible individual who becomes infected can therefore rapidly spread the pathogen to other susceptible individuals, requiring them to take antibiotics to clear the infection. Widespread antibiotic use, however, drives an increase in antibiotic-resistant bacteria, which can rapidly spread resistance around the globe. (b) If large proportions of the population have been immunized against a pathogen, disease and the spread of disease are prevented. This results in much lower overall antibiotic use in the population, thus stemming the further development of antibiotic resistance.

of antibiotics, including most oral agents used for common treatment of urinary tract infections. On a global basis, M. tuberculosis has the highest burden of infected individuals of any single infectious disease, with approximately 2 billion people infected and with a devastatingly high prevalence in developing countries ${ }^{18}$. Tuberculosis (TB) is exacerbated in regions where there is an overlap in HIV infection. This can be due to reactivation of latent TB disease or rapid progression of primary infection due to waning $\mathrm{T}$ cell immunity. In addition, TB drug resistance that already is substantial in some countries (Table 1) further compounds treatment efforts and leads to lower cure rates and longer time on therapy.

\section{The power of vaccines to address AMR}

Reliance on antibacterial antibiotics has been exacerbated by the development of broad-spectrum antibiotics that can be applied to treat infections before diagnosis of the specific pathogen. In many cases, a broad-spectrum antibiotic is used for relatively minor infections even though the pathogen is never identified, or a broad-spectrum antibiotic may also be used in cases where the etiology of the infection may not have been a bacterial pathogen. While broad-spectrum antibiotics are straightforward to deliver, they have been a major driver for bacterial AMR. There has been a subsequent drive in research and development efforts to develop treatment approaches that are more pathogen specific using small molecules and monoclonal antibodies. However, these more specific treatments require the pathogen to be identified before treatment, which can take several days and thus poses risks to the patient.
This 'identification first' paradigm differs significantly from the current paradigm of treating once an infection is suspected and changing the antibiotic if the patient's condition does not improve. Prophylactic vaccines, however, represent a change in the infectious disease paradigm, as they prevent the disease from occurring in the first place and are also species specific.

Vaccines work by training the immune system to recognize and respond to a pathogen by mounting a rapid and effective immune defense, preventing the establishment of an infection/disease or decreasing disease severity ${ }^{19}$. Many vaccines also protect unvaccinated individuals or subjects who cannot be vaccinated in the population through a process called herd immunity (Fig. 2). Herd immunity is an added benefit when vaccines are broadly administered in the population for which the vaccine is licensed; not only are the vaccinated individuals protected from the disease, but, by preventing the disease, transmission of the pathogen is interrupted to groups of individuals for whom the vaccine is not licensed, those that cannot be immunized or individuals who are immunocompromised (i.e., patients with cancer undergoing chemotherapy $)^{19,20}$. Herd immunity results in protection against infection of much larger numbers of individuals than those who have been vaccinated in the community. Some of the most successful vaccines, like the conjugate vaccines that protect against Hib and S. pneumoniae discussed below, lead to highly effective herd immunity as they also prevent pathogen colonization in individuals who are vaccinated.

Eradication of disease or sustained global disease reduction often leads to an underappreciation of the power of vaccines to protect a 


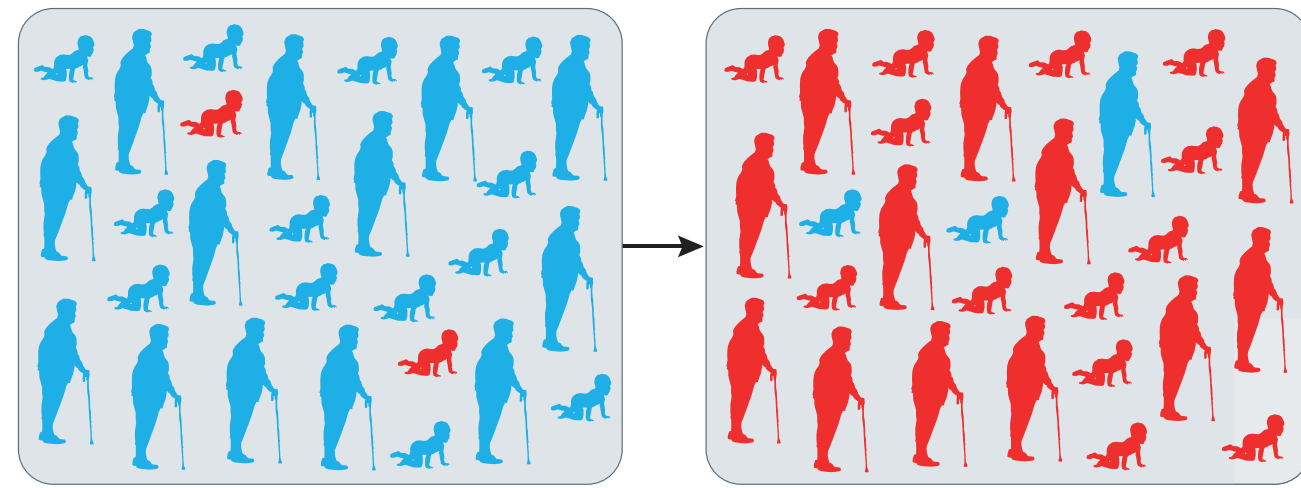

b

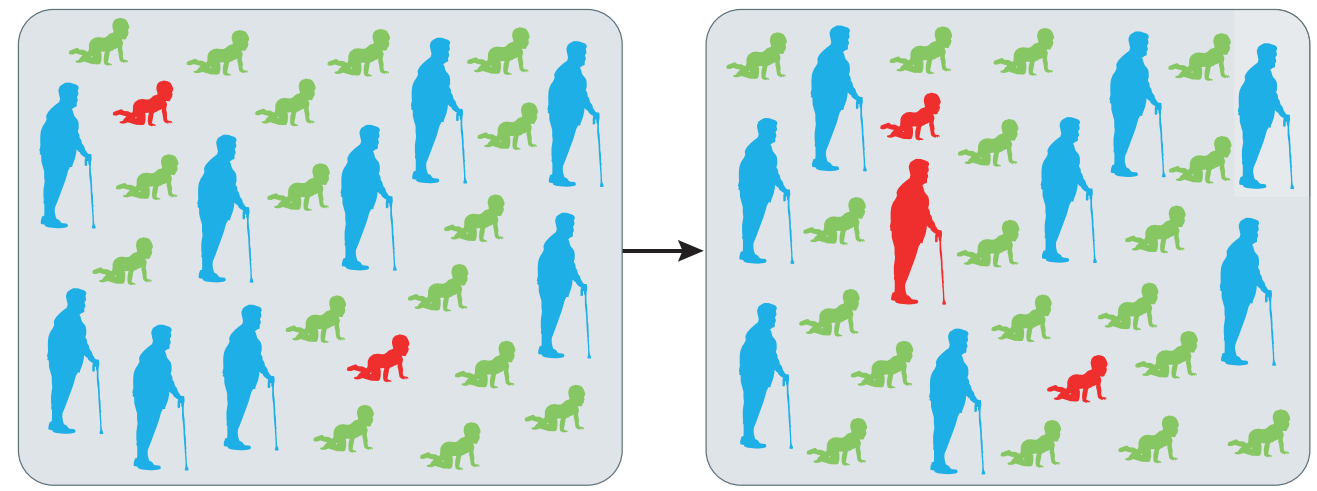

Figure 2 The principle of herd immunity. (a) No immunization: the bacterial pathogen spreads uninhibited in a susceptible population. Infected individuals in the population spread disease to susceptible individuals, and over time a large proportion of the population is infected. (b) With immunization: broad immunization in the population interrupts transmission of the bacterial pathogen, and susceptible individuals, including those that have not or cannot be immunized (i.e., immunocompromised individuals), are now protected from disease.

large proportion of the world's population from disease and death. In fact, immunization prevents millions of deaths every year, and this number could increase further as global vaccination rates continue to improve $^{21}$. For example, the current global coverage for the pneumococcal conjugate vaccine is estimated to be only $37 \%$ and any increase in vaccination coverage would further decrease disease rates and use of antibiotics (http://www.who.int/mediacentre/factsheets/fs378/en/). Unbeknownst to many, however, vaccines also have an excellent track record in reducing AMR by preventing the spread of disease and, thus, antibiotic use. The consequent reduced use of antibiotics then results indirectly in reduction of AMR.

Prominent examples of how vaccines can assist in the fight against AMR are described below and include S. pneumoniae conjugate and Hib conjugate vaccines. The implementation of viral vaccines such as those to prevent influenza (discussed below) and varicella also have a proven role in reducing antibiotic use $\mathrm{e}^{22}$.

\section{Vaccines proven to reduce AMR}

Haemophilus influenzae type b vaccines. Hib conjugate vaccines were the first effective bacterial vaccines that not only showed high efficacy in preventing invasive disease in immunized infants and protecting older children by herd immunity, but also significantly reduced antibiotic use and hence the development of antibiotic resistance. Before Hib conjugate vaccines were first introduced in the late 1980s in the United States and then globally, Hib was a devastating pathogen in infants and young children. At that time, incidence rates of Hib disease (including bacterial meningitis and other invasive diseases) in children $<5$ years of age ranged from 3.5 to 601 cases per 100,000 in many countries of the world ${ }^{23}$. In addition, a steady increase in Hib $\beta$-lactam resistance was observed since the early $1970 \mathrm{~s}^{24}$, mediated by bacterial expression of $\beta$-lactamases and/or, to a lesser extent, modified penicillin-binding proteins ${ }^{24}$. For example, one global surveillance study carried out from 1999 to 2000 found that $16.6 \%$ of all Hib strains worldwide were $\beta$-lactamase positive, with large variation between countries ${ }^{25}$.

Fortunately, the development and deployment of effective Hib conjugate vaccines has turned the tide against antibiotic resistance ${ }^{26}$. Conjugate vaccines against Hib developed in the 1960s consisted of the Hib polysaccharide capsule conjugated to carrier proteins. Disease cases dropped precipitously after the introduction of routine use of $\mathrm{Hib}$ conjugate vaccines ${ }^{27}$. In addition, decreases in nasopharyngeal carriage after Hib vaccination were notable, and this was a prerequisite for herd immunity and extended protection of unvaccinated populations. For example, after Hib conjugate vaccine introduction and recommendation in Canada in 1988, rates of Hib disease dropped from 2.6 cases per 100,000 (1986-1987) to 0.08 cases per 100,000 in (2011-2015; ref. 28). Similarly, vaccine introduction in the UK in 1992 resulted in near elimination of $\mathrm{Hib}$ disease in children $<5$ years of age within just a few years of vaccine introduction, and comparable successes were observed globally ${ }^{29}$. Shortly after Hib conjugate vaccine introductions, a significant decrease in $\beta$-lactamase-positive strains was also observed ${ }^{30}$, although in some countries ( such as Japan ${ }^{31}$ ) the prevalence of these resistant strains may have been decreasing before vaccine implementation.

Pneumococcal vaccines. S. pneumoniae continues to be a leading global cause of serious illness among unvaccinated children and adults. In 2005, the WHO estimated that 1.6 million deaths were caused by this pathogen 


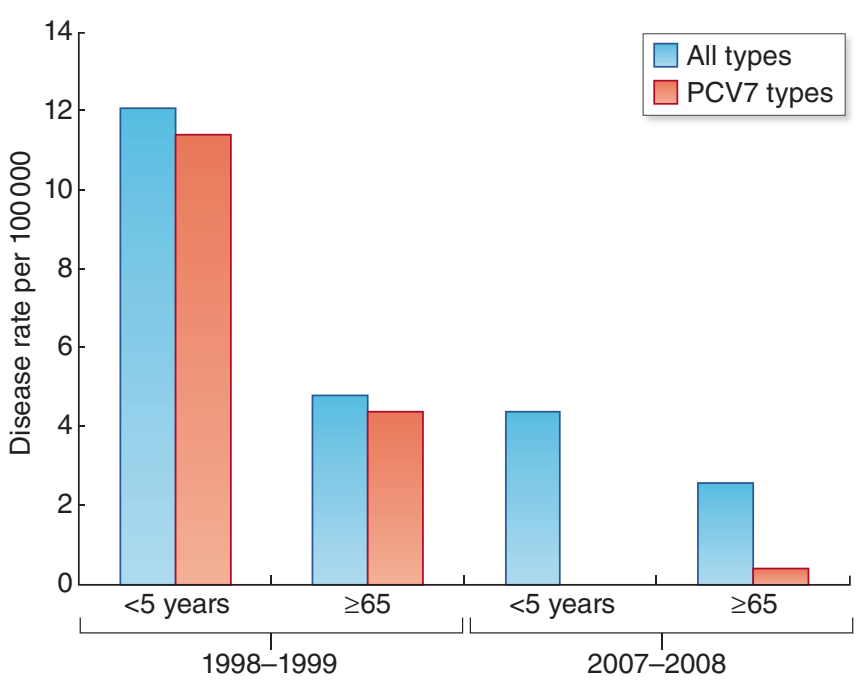

Figure 3 Reduction of AMR after broad rollout of pneumococcal conjugate vaccine. The impact of PCV7 on disease rates of $S$. pneumoniae strains not susceptible to penicillin in children under 5 years of age and the indirect positive effect (herd effect) on the elderly ( $\geq 65$ years old) is shown comparing incidence in the time frame before PCV7 implementation (1998-1999) to that in the time frame after vaccine implementation $(2007-2008)^{34}$.

annually (http://www.who.int/ith/diseases/pneumococcal/en/). In the 1990 s, before the introduction of a 7 -valent pneumococcal conjugate vaccine (PCV7) in children, 63,000 cases of invasive pneumococcal disease caused by vaccine serotype (serotypes covered by PCV7) and non-vaccine serotype (serotypes not covered by PCV7) pneumococcus occurred each year in the United States ${ }^{32}$. Importantly, at this time, resistance to penicillin and other classes of antibiotics also spread in $S$. pneumoniae in the United States, with invasive pneumococci becoming resistant to three or more drug classes $^{33,34}$.

Similar to Hib vaccines, pneumococcal polysaccharide conjugate vaccines have been a tremendous success, with $>90 \%$ efficacy against invasive pneumococcal disease (IPD) observed in the primary target populations of children $<5$ years of age ${ }^{35}$. Not only was disease prevented, but significant reductions in bacterial colonization in US children were observed, contributing to herd immunity in individuals initially not targeted for routine immunization, in particular, in adults ${ }^{35}$.

Only seven years after the introduction of PCV7 in 2000, 211,000 cases of IPD caused by the seven serotypes covered by PCV7 (including antibiotic-resistant strains) were prevented not only in children but in individuals of all ages ${ }^{34,35}$ (Fig. 3). Second-generation pneumococcal conjugate vaccines with extended serotype coverage such as PCV10 (10 valent) and PCV13 (13 valent) have increased direct protection and herd immunity to the pneumococcal serotypes covered by these vaccines with similar efficacy ${ }^{36,37}$. In addition, PCV13 reduced antibiotic use, and the prevalence of strains not susceptible to antibiotics decreased

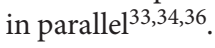

\section{Preventing AMR using viral vaccines}

Influenza vaccines. Influenza vaccines not only prevent influenza infections and disease, but also decrease the likelihood of secondary bacterial infections, such as pneumonia and otitis media ${ }^{38}$. There are two major mechanisms by which even moderately effective influenza vaccines reduce antibiotic use. First, they prevent secondary bacterial infections and thus reduce the use of antibiotics to treat such infections. In one study conducted in Turkey, the incidence of acute otitis media was significantly reduced by $50.9 \%$ in children receiving influenza vaccine as compared to unvaccinated controls and, by inference, antibiotic use was similarly reduced in the vaccinated children ${ }^{39}$. A study conducted in Canada demonstrated convincingly that the rate at which influenza-associated antibiotics were prescribed was $\sim 64 \%$ lower after the introduction of universal influenza vaccination in Ontario than in other Canadian provinces that limited the use of influenza vaccines to at-risk situations ${ }^{11}$.

The second benefit of influenza vaccines for AMR reduction is the prevention of inappropriate antibiotic prescriptions for respiratory tract infections caused by viral pathogens. For example, in the United States, half of all antibiotic prescriptions are inappropriately written for respiratory illnesses associated with pathogens such as influenza that are not susceptible to antibiotics ${ }^{8}$. These examples illustrate the important role viral vaccines can play in reducing antibiotics use that drives bacterial resistance. Further research could deliver vaccines that address other viral diseases as well as improve upon existing influenza vaccines to provide higher efficacy in older adults and young children and increase the breadth of vaccine efficacy to an extended repertoire of viral strains.

Respiratory syncytial virus vaccines. RSV is the leading cause of bronchiolitis and viral pneumonia in infants and can lead to fatal respiratory distress, especially in very young infants, in infants with underlying cardio-pulmonary disease or in the absence of effective healthcare systems. Globally, by two years of age, nearly everyone has been infected with RSV ${ }^{40}$. Worldwide, RSV kills approximately 66,000-199,000 infants annually, with the vast majority of these deaths occurring in developing countries ${ }^{41}$. In the United States, RSV is the leading cause of infant hospitalization, with approximately 50,000 hospitalizations of children 12 months of age and younger annually ${ }^{42}$. RSV is another example of a viral disease that, in a similar manner to influenza, contributes to AMR, given that antibiotics are often prescribed inappropriately for treatment of symptoms of a viral disease before the etiological agent for the infection has been determined. In addition, RSV infection is associated with bacterial infections such as otitis media and pneumonia requiring the use of antibiotics, hence increasing pressure for drug resistance ${ }^{43}$.

RSV also causes respiratory illness in older adults and is responsible for approximately 100,000 hospitalizations and 5,000 deaths annually in the United States in adults 65 years of age and older ${ }^{44-47}$. In the United States, RSV disease rates in older adults are approximately half those of influenza ${ }^{45}$. Like influenza, RSV infection is associated with the development of bacterial superinfections ${ }^{48-50}$, and animal model studies have elucidated potential mechanisms for lethal synergy between the two infections ${ }^{51}$. Thus, RSV vaccines have the potential to further reduce the inappropriate use of antibiotics in RSV infections, prevent bacterial superinfections and facilitate appropriate antibiotic use in both children and the elderly.

RSV vaccine development has been riddled with many failures, mostly because of an incomplete understanding of the pathobiology of this important viral pathogen and technical difficulties in producing an effective vaccine. It is well established that the trimeric RSV F surface glycoprotein is the primary target of protective neutralizing antibodies and mediates the fusion of the viral membrane with the membrane of the host cell. Vaccine development has historically focused on the post-fusion form of RSV F, and this approach has failed multiple times in phase 3 studies in adults ${ }^{52-54}$. More potent RSV neutralizing antibodies are elicited to the pre-fusion F protein ${ }^{55}$, and several promising vaccine candidates based on pre-fusion $\mathrm{F}$ are currently under development. Given the medical need for an RSV vaccine, such vaccines, if successfully developed, will not just prevent the viral disease itself, but will also curtail the inappropriate and 
Table 2 Vaccine candidates in clinical development with the potential to prevent diseases caused by pathogens highlighted in this review

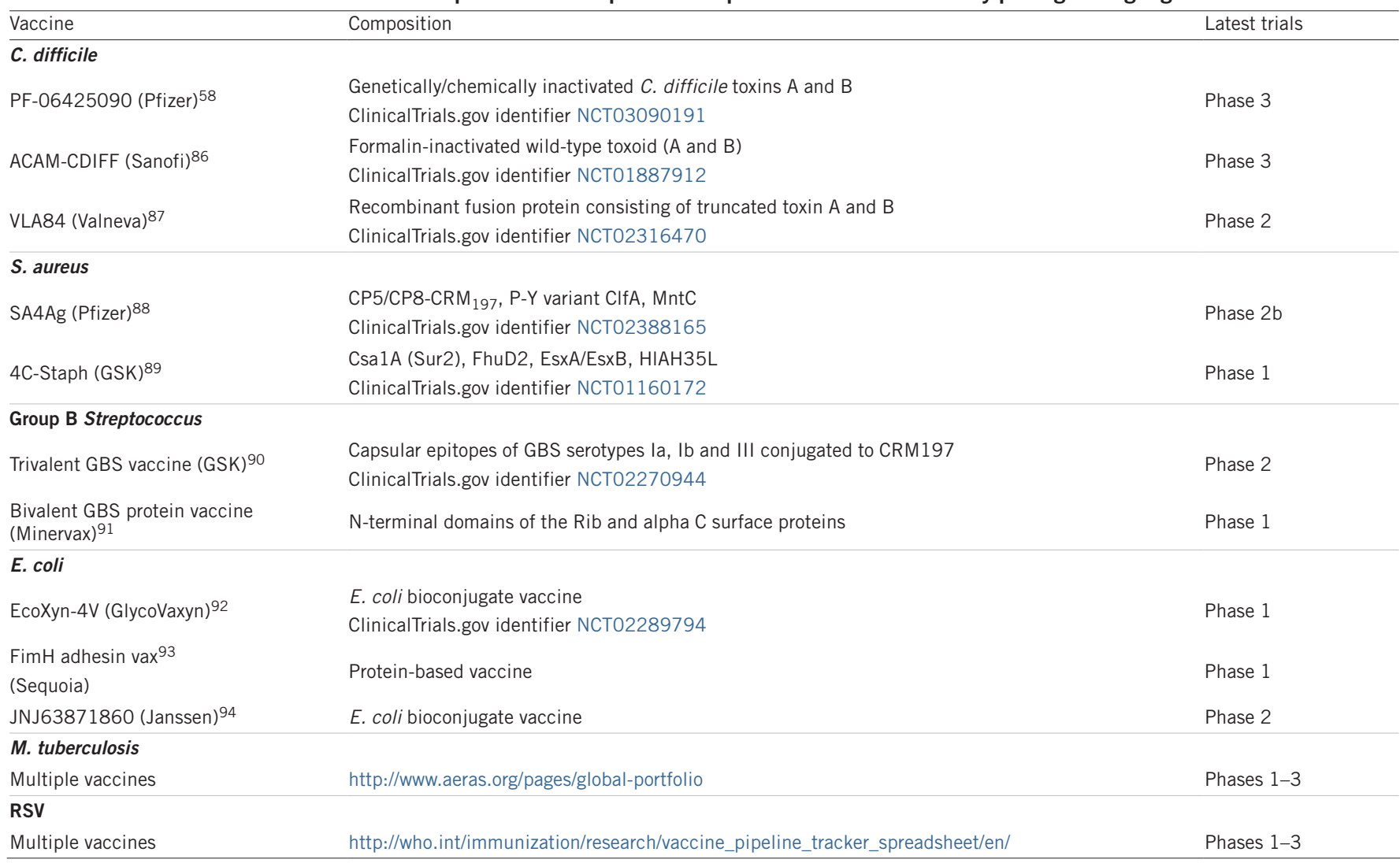

appropriate (to treat bacterial superinfections) use of antibiotics and the resulting development of AMR.

\section{Current vaccines under development with potential to reduce AMR}

Vaccine development is a lengthy process; thus, careful consideration has to take place with regard to which vaccines should and actually can be developed. Before attempting to develop vaccines, a number of requirements should be met. Considerations include foremost the extent of the unmet medical need, i.e., the prevalence of the disease. Furthermore, it is necessary to have a scientific understanding of the immune responses required to protect against the pathogen so that the vaccine can be appropriately configured. In addition, there needs to be the technical knowhow to design and produce an appropriate vaccine with the desired stability characteristics. Another hurdle to overcome is the identification of a patient population with high enough disease incidence to test a vaccine under development.

Usually, most of these requirements have to be met to ensure successful vaccine development, which explains why not every bacterial AMR pathogen is a candidate for vaccine development. Current vaccine research and development focuses on many of the organisms with increased antibiotic resistance or associated with high antibiotic use, such as those listed in Table 2 . In the following sections, we discuss select examples of vaccines under development that have met all the requirements described above, that in every case target pathogens with demonstrated propensity to acquire antibiotic resistance and that thus hold promise in further reducing AMR.

Clostridium difficile vaccines. C. difficile, a Gram-positive anaerobic, spore-forming bacillus, is the main cause of nosocomial infectious diarrhea in industrialized countries. It accounts for $20-30 \%$ of cases of diarrhea that develop after antibiotic use and associated disruption of the normal gut microflora and is the most commonly recognized cause of infectious diarrhea in healthcare settings ${ }^{56}$. C. difficile is carried in approximately $1-3 \%$ of healthy adults and approximately $16-35 \%$ of hospital inpatients on antibiotic treatment. Fifty percent or more of hospital patients colonized by C. difficile are asymptomatic carriers ${ }^{56}$.

In the United States alone, approximately half a million cases of $C$. difficile infection (CDI) are diagnosed annually, with 29,000 associated deaths. Given this burden of CDI and the rapid global spread of epidemic and antibiotic-resistant strains, the CDC has classified C. difficile as an urgent threat ${ }^{16}$. Once an infection occurs, the patient is often caught in a vicious cycle in which CDI recurs, requiring additional antibiotic use. Thus, vaccine development is of high priority given the large unmet medical need for this type of infection.

$\mathrm{CDI}$ is mediated by toxins that can be neutralized by toxin-neutralizing monoclonal or polyclonal vaccine-elicited antibodies ${ }^{57,58}$. The clear mechanism of action/pathogenesis of this toxin-based disease and an understanding that vaccine-induced generation of toxin-neutralizing antibodies has proven successful for a number of other toxin-based diseases (such as tetanus, diphtheria and anthrax) further provided the rationale that a vaccine for protection against $C$. difficile should be technically feasible. Having met essentially all of the vaccine development requirements listed above, two $C$. difficile vaccines are now in phase 3 clinical development ${ }^{59,60}$. Because CDI incidence increases with age, the vaccines are being developed to protect adults 50 years and older. Once licensed and universally recommended, they would provide additional highly effective tools in preventing AMR, as vaccinated individuals should not contract CDI, thus reducing the spread of spores and bacteria, the associated antibiotic use and the generation of MDR bacterial species. 


\section{REVIEW}

Staphylococcus aureus vaccines. S. aureus is a major cause of invasive disease including bacteremia, infectious endocarditis, osteomyelitis, pneumonia, and various skin and soft tissue infections in hospital and community settings. Surgical site infections (SSIs) account for over $40 \%$ of nosocomial infections in surgical patients. These infections currently have an estimated annual incidence of 300,000-500,000 in the United States ${ }^{61,62}$, a large percentage of which are caused by S. aureus $s^{63}$. Infections can be complicated by bacterial resistance to $\beta$-lactams and other classes of antibiotics. Infections caused by MRSA are harder to treat than those due to methicillin-sensitive strains and are associated with prolonged hospital stays and increased morbidity and mortality. MRSA contributes to over 70,000 cases of invasive disease per year in the United States alone. Both the CDC and WHO have recently placed MRSA on their AMR watchlists, highlighting the significance of AMR in S. aureus.

S. aureus has the propensity to develop resistance even to newly introduced antibiotics, such as linezolid, a drug that inhibits protein synthesis in Gram-positive bacteria introduced in 2000, and daptomycin, a drug that disrupts the membrane of Gram-positive bacteria introduced in 2003 (ref. 64). S. aureus glycopeptide resistance is currently a source of concern, as this class of antibiotics, including vancomycin, is one of the main recourses for combating infections caused by MRSA. Reduced susceptibility to vancomycin was first described in 1996 in $\operatorname{Japan}^{65}$. Thereafter, an $S$. aureus isolate with acquired heterogeneous resistance to vancomycin was characterized ${ }^{66}$. Thus far, strains of MRSA with acquired resistance to vancomycin are rare; however, widespread and often uncontrolled empirical vancomycin use, particularly during elective surgeries, could further drive resistance. This has already been documented in the emergence of vancomycin-resistant enterococci and Vancomycin-intermediate Staphylococcus aureus (VISA) ${ }^{12,67,68}$. Given the disease burden and adaptability of $S$. aureus to develop resistance to many classes of antibiotics, a strong rationale exists to develop effective vaccines to protect individuals against this species and to reduce AMR.

A more complete understanding of $S$. aureus has yielded several new candidate $S$. aureus vaccines currently in development ${ }^{69,70}$. The most advanced is a 4 -antigen $S$. aureus vaccine candidate (SA4Ag) that is being evaluated in a phase $2 / 3$ efficacy study for prevention of invasive S. aureus disease in patients undergoing elective spinal surgery. SA4Ag was shown to be safe and well tolerated in early-stage clinical trials, and was found to induce high levels of bacteria-killing antibodies in healthy adults, leading to a fast-track designation by the US Food and Drug Administration (FDA). In addition, a separate 4-component S. aureus vaccine (4C-Staph) formulated with a Toll-like receptor 7 (TLR7)dependent adjuvant is being tested in phase 1 trials. Initially, these vaccines are being developed for prevention of invasive $S$. aureus infections in patients undergoing elective surgeries.

Group B Streptococcus vaccines. Streptococcus agalactiae, also known as group B Streptococcus (GBS), is an encapsulated Gram-positive bacterium that asymptomatically colonizes the vagina (women) and rectum (women and men) of approximately $25 \%$ of the population and can cause serious invasive infections, including pneumonia, meningitis, bacteremia and sepsis, across all age groups. Pregnant women and their babies are vulnerable to disease resulting from this organism, as asymptomatic maternal carriage is a prime risk factor for diseases in the mother, such as urinary tract infections, endometritis, chorioamnionitis, sepsis, preterm labor and still birth, and in the baby. Infant disease as a result of GBS infection presenting within the first 90 days of life is particularly devastating.

In the United States and some other high-income countries, GBSrelated disease incidence has been reduced by screening pregnant women for GBS recto-vaginal colonization and then treating carriers with antibiotics during labor, a process known as intrapartum antibiotic prophylaxis (IAP). In the United States, approximately $25 \%$ of pregnant women are reported to undergo IAP, and even higher rates are seen in other countries such as Canada ${ }^{71}$. Although resistance to penicillin is low, approximately $10 \%$ of the maternal population report penicillin allergies, leading to the administration of macrolide antibiotics instead. However, the prevalence of macrolide resistance in GBS has been rising in recent years, ranging from $15 \%$ in the United States to over $60 \%$ in Asia, leaving physicians to use vancomycin for mothers with macrolideresistant GBS. In addition, the routine application of IAP (at least in the United States for $\sim 30 \%$ of pregnancies) poses a dilemma because antibiotic exposure during late-stage pregnancy and early in life has recently been linked to a range of adverse health outcomes later in life, which could be avoided with the implementation of an effective vaccine ${ }^{72}$.

GBS capsular polysaccharide conjugate vaccines under development (Table 2) are designed to induce functional antibodies when administered as maternal vaccines to pregnant women. These antibodies are then transferred to their babies during the last trimester of pregnancy and provide a potential basis to protect them from GBS disease. Current vaccine development is focused on capsular polysaccharide conjugate vaccines (Table 2), as capsular-serotype-specific antibodies have been shown to kill GBS via complement-mediated phagocytosis.

Vaccines to prevent Enterobacteriaceae infections. As described above, Enterobacteriaceae are on the WHO list of priority pathogens given their antibiotic resistance ${ }^{73}$. Of the organisms in this family, E. coli and $K$. pneumoniae pose the greatest concerns because they have high levels of resistance to third-generation cephalosporins and extended-spectrum $\beta$-lactam antibiotics. E. coli infections are also associated with high levels of fluoroquinolone resistance, and K. pneumoniae is highly resistant to carbapenems. Both species are part of the normal flora in the intestine in humans and animals. E. coli is the most frequent cause of urinary tract infections (including pyelonephritis acquired outside of a hospital setting (community acquired) and inside a hospital (nosocomial)). It is also the most frequent cause of bloodstream infection at all ages and is associated with intra-abdominal infections such as peritonitis. E. coli is a cause of meningitis in neonates and is one of the leading causative agents of foodborne infections worldwide. K. pneumoniae poses a similar problem; however, it tends to infect more vulnerable individuals, including preterm infants and individuals with impaired immune systems, diabetes or alcohol abuse issues, as well as those receiving advanced medical care.

MDR Enterobacteriaceae have achieved greater attention recently owing to the rapid global spread of an NDM- 1 metallo- $\beta$-lactamaseproducing strain. This strain illustrates how the world is increasingly connected, given that it was isolated in patients in Europe who had previously traveled to India for surgical proceedures ${ }^{74,75}$. Today, NDM-1 is present worldwide and has also spread to other Gram-negative species, rendering them resistant to all agents except colistin and tigecycline. With the introduction of MCR-1 plasmid-1 into E. coli isolates conferring resistance to the last-line antibacterial colistin, there is concern that strains containing both MCR-1 and NDM-1 may emerge and turn routine outpatient urinary tract infections untreatable ${ }^{76}$.

Some Gram-negative organisms present clinical development challenges with regard to identifying an appropriate patient population with high enough disease incidence to perform prophylactic vaccine clinical studies capable of producing the data to satisfy regulatory approval and recommendation. However, for a pathogen such as E. coli, such patient populations can be identified and promising vaccine approaches focusing on lipopolysaccharides (or $\mathrm{O}$ antigens) are being evaluated clinically 
$(\text { Table } 2)^{77,78}$. Additional approaches are in preclinical or early clinical stages of development ${ }^{79,80}$.

Other Gram-negative pathogens. Three additional Gram-negative pathogens that warrant discussion are carbapenem-resistant Acinetobacter baumannii, carbapenem-resistant Pseudomonas aeruginosa and thirdgeneration cephalosporin-resistant, fluoroquinolone-resistant Neisseria gonorrhoeae. All three are included on the WHO priority pathogen list. $A$. baumannii and $P$. aeruginosa infections are both associated with healthcare settings and, although they do not cause high rates of infection, they are characterized by high levels of antibiotic resistance and associated morbidity; there are no vaccines currently in development for these pathogens. A vaccine for N. gonorrhoeae would need to improve upon the ineffective human adaptive immune response to wild-type infection that allows for multiple reinfections. A further obstacle to vaccine development efforts is the lack of robust animal models for this species of Gram-negative bacteria. Antigenic variation also confounds $N$. gonorrhoeae vaccine discovery, as was observed in the case of a pilinbased vaccine trial $^{81}$.

Mycobacterium tuberculosis vaccines. TB has the highest global burden of any single infectious disease, with almost 2 billion people latently infected and an associated approximately 1.8 million deaths annually ${ }^{82}$. Co-infection with tuberculosis in individuals with HIV is estimated to occur in 15 million people and, given the progressive $\mathrm{T}$ cell dysfunction in HIV, TB can occur early on in infection and be a significant cause of morbidity and mortality in HIV-infected people. Globally, approximately $5 \%$ of the 10.4 million newly diagnosed TB cases were MDR in 2015, and AMR leads to longer treatment regimens, prolonged periods of contagiousness and higher mortality in those affected with TB. Thus, the development of vaccines may have a profound impact on disease morbidity and mortality and assist in stemming AMR.

Vaccines could induce protection against TB by a number of mechanisms, including by preventing the initial infection, reducing the reactivation of latent infection or potentiating immunity to $\mathrm{TB}$ in immunocompromised hosts. The Bacillus Calmette-Guérin (BCG) vaccine, in use for almost 100 years, is composed of an attenuated strain of Mycobacterium bovis and is used to vaccinate $>90 \%$ of newborns in endemic countries. This vaccination program is based on studies that have demonstrated that vaccination results in a reduction of disseminated disease and mortality in the youngest children. Readministering BCG does not provide additional protection after a childhood dose, and BCG will not prevent the reactivation of latent TB to pulmonary disease in the nearly one-third of the human population who are already infected and at risk. Given these shortcomings of BCG, a number of more modern vaccine approaches are being pursued ${ }^{18,83}$.

Examples of such approaches include elicitation of better priming with genetically modified BCG, use of other whole-cell mycobacterial preparations or alternatively developing subunit vaccines. The subunit vaccine that has advanced furthest in clinical trials used a vectored approach with modified vaccinia virus Ankara expressing recombinant antigen 85A (MVA85A). However, the vaccine failed to provide protection against TB infection or prevent progression to active disease in infants in South Africa or in HIV-1-infected adults in South Africa and Senegal. An inactivated whole-cell Mycobacterium vaccae vaccine administered with five intradermal doses had a vaccine efficacy of $39 \%$ (i.e., it resulted in $39 \%$ less TB cases than control) that reached statistical significance in preventing culture-confirmed $\mathrm{TB}^{84}$. As discussed above, $\mathrm{TB}$ is an example of an infectious disease where the vaccine development requirements have largely not been met. While there is a clear medical need for a vaccine, future scientific advances to fully understand the mechanisms of immune protection and to develop new technologies to induce the desired responses are needed to address AMR issues associated with this pathogen.

\section{Barriers to maximizing licensed vaccine use}

Vaccines have been very effective in reducing incidence of infectious diseases and use of antibiotics and AMR in some relevant pathogens such as S. pneumoniae and Hib; yet, there remain a number of hurdles to global access, implementation and use. While vaccine access in developed countries has reached high rates of population coverage for vaccines such as Hib and pneumococcal conjugate vaccines, global implementation is dramatically lower, with coverage rates of only $19-45 \%$ of the population $^{20}$. Global coverage could be improved by simultaneous licensure in developed and developing countries, faster rollout in countries with the largest birth cohorts such as China and India, improving logistics to bring vaccines to remote locations, increasing funding and lowering population reticence to vaccination. Regulatory oversight of clinical studies and other studies often required by regulatory agencies after vaccine licensure (postmarketing commitment studies) provide very robust vaccine benefit-risk assessments; however, these usually extensive data sets are at times not fully taken into consideration by vaccine-recommending bodies in many countries; thus, widespread access, reimbursement of costs associated with vaccines and their administration by healthcare providers, and use of vaccines may be delayed.

Advocacy on the safe and effective use of vaccines for medical professionals and the general public is provided by academic and public-health institutions and is complemented by organizations such as Vaccines Europe, a European Union (EU)-wide vaccine industry stakeholder group of major innovative research and development-based vaccine companies. Vaccines Europe provides information to the public regarding the vaccine innovation and manufacturing process and highlights evidence meticulously developed on the safe and effective use of vaccines. However, even in developed countries such as the United States and in Europe, coverage with some vaccines such as influenza vaccines remains suboptimal and could be greatly improved for licensed vaccines by stronger recommendations and more education on the use of these vaccines. Optimal implementation and use could have a profound impact on disease burden and the inappropriate use of antibiotics.

\section{Future prospects}

At a time when the global community is just starting to mobilize resources to develop therapeutics to counteract AMR, ongoing vaccine research and development programs have the potential to address AMR prevention by decreasing overall antibiotic prescription pressure, especially when coupled with educational interventions to limit antibiotic use. Much remains to be done to provide global access to already licensed vaccines, and prospects are favorable for additional vaccine approaches to continue to provide global public-health benefits. To provide maximal public-health benefits, there must be efforts to steadily increase vaccine coverage as well as complete study and licensure proceedings for new vaccines addressing important bacterial and viral pathogens for which vaccines did not previously exist.

Newer vaccines under development such as vaccines to prevent C. difficile or S. aureus infection, pneumococcal conjugate vaccines with extended serotype coverage or vaccines to prevent infections with Gramnegative bacteria hold a profound promise to not only address these lifethreatening diseases but also help to further curb antibiotic use and thereby prevent AMR. In addition, there is no limit to the number of vaccines that can be given to an individual, as humans are exposed on a continuous basis to a myriad of infectious disease agents and the immune system has been developed to deal with an almost infinite number of pathogens. 


\section{REVIEW}

The prospects for new antibiotics and classes of antibiotics are better than ever today, given the increased scientific understanding of bacterial pathology, and will benefit from novel public- and privatesector collaborations through funding from the European Innovative Medicines Initiative and a newly formed US funding entity, CARB X (http://www.imi.europa.eu/content/nd4bb and https://www.bu.edu/ law/faculty-scholarship/carb-x/). AMR mitigation efforts will benefit from a combined effort to prevent infections with new vaccines and develop new therapeutics for infections that were not prevented. As a note of caution, just as was observed in the context of research and development efforts to discover new antibacterial drugs, vaccine research and development organizations have seen dramatic consolidation over the last decade, hence limiting the pool of experienced human talent required and available to deliver on the scale needed for complex clinical and basic science programs ${ }^{95}$. The incentive to develop new vaccines would benefit from innovation in regulatory sciences to improve upon the speed of future vaccine development and to bring new preventative measures for AMR to the clinic sooner. In parallel, sustained investments in developing human talent would provide an experienced workforce to innovate across research and development. These investments are needed to provide future leadership to thoughtfully advance vaccines into the clinic. Additionally, the importance of maintaining and creating new markets for vaccines cannot be overstated. This is vital to provide incentives to vaccine companies to develop vaccines that are of public interest but may not be commercially viable. Given the importance of vaccines in reducing AMR, not addressing these trends could become a matter of national concern across the globe.

\section{ACKNOWLEDGEMENTS}

The authors acknowledge the editorial contributions of S. Vuocolo and M. Hopps.

\section{COMPETING FINANCIAL INTERESTS}

The author declares competing financial interests: details are available in the online version of the paper.

Reprints and permissions information is available online at http://www.nature.com/ reprints/index.html. Publisher's note: Springer Nature remains neutral with regard to jurisdictional claims in published maps and institutional affiliations.

1. Levy, S.B. \& Marshall, B. Antibacterial resistance worldwide: causes, challenges and responses. Nat. Med. 10 (Suppl.), S122-S129 (2004).

2. Coates, A.R.M. Antibiotic Resistance (Springer Science \& Business Media, 2012).

3. Bronzwaer, S.L. et al. A European study on the relationship between antimicrobial use and antimicrobial resistance. Emerg. Infect. Dis. 8, 278-282 (2002).

4. Tängdén, T., Eriksson, B.M., Melhus, A., Svennblad, B. \& Cars, O. Radical reduction of cephalosporin use at a tertiary hospital after educational antibiotic intervention during an outbreak of extended-spectrum $\beta$-lactamase-producing Klebsiella pneumoniae. J. Antimicrob. Chemother. 66, 1161-1167 (2011).

5. O'Neill, J. Tackling Drug-Resistant Infections Globally: Final Report and Recommendations (The Review On Antimicrobial Resistance, 2015).

6. Ventola, C.L. The antibiotic resistance crisis: part 1: causes and threats. $P \& T 40$ 277-283 (2015)

7. Goff, D.A. et al. A global call from five countries to collaborate in antibiotic stewardship: united we succeed, divided we might fail. Lancet Infect. Dis. 17, e56-e63 (2017).

8. Fleming-Dutra, K.E. et al. Prevalence of inappropriate antibiotic prescriptions among US ambulatory care visits, 2010-2011. J. Am. Med. Assoc. 315, 1864-1873 (2016).

9. Landers, T.F., Cohen, B., Wittum, T.E. \& Larson, E.L. A review of antibiotic use in food animals: perspective, policy, and potential. Public Health Rep. 127, 4-22 (2012).

10. Lipsitch, M. \& Siber, G.R. How can vaccines contribute to solving the antimicrobial resistance problem? MBio 7, e00428-16 (2016).

11. Kwong, J.C., Maaten, S., Upshur, R.E.G., Patrick, D.M. \& Marra, F. The effect of universal influenza immunization on antibiotic prescriptions: an ecological study. Clin. Infect. Dis. 49, 750-756 (2009).

12. Neu, H.C. The crisis in antibiotic resistance. Science 257, 1064-1073 (1992)

13. Breiman, R.F., Butler, J.C., Tenover, F.C., Elliott, J.A. \& Facklam, R.R. Emergence of drug-resistant pneumococcal infections in the United States. J. Am. Med. Assoc. 271, 1831-1835 (1994)

14. Sieradzki, K., Roberts, R.B., Haber, S.W. \& Tomasz, A. The development of vancomycin resistance in a patient with methicillin-resistant Staphylococcus aureus infection. N. Engl. J. Med. 340, 517-523 (1999).
15. Peng, Z. et al. Update on antimicrobial resistance in Clostridium difficile: resistance mechanisms and antimicrobial susceptibility testing. J. Clin. Microbiol. 55, 19982008 (2017).

16. He, M. et al. Emergence and global spread of epidemic healthcare-associated Clostridium difficile. Nat. Genet. 45, 109-113 (2013).

17. Weinstein, R.A., Gaynes, R., Edwards, J.R. \& National Nosocomial Infections Surveillance System. Overview of nosocomial infections caused by gram-negative bacilli. Nephrol. Dial. Transplant. 41, 848-854 (2005).

18. Raviglione, M. et al. Scaling up interventions to achieve global tuberculosis control: progress and new developments. Lancet 379, 1902-1913 (2012).

19. Fine, P.E. Herd immunity: history, theory, practice. Epidemiol. Rev. 15, 265-302 (1993).

20. Greenwood, B. The contribution of vaccination to global health: past, present and future. Phil. Trans. R. Soc. Lond. B 369, 20130433 (2014).

21. Klatt, T.E. \& Hopp, E. Effect of a best-practice alert on the rate of influenza vaccination of pregnant women. Obstet. Gynecol. 119, 301-305 (2012)

22. Bozzola, E. et al. Varicella skin complications in childhood: a case series and a systematic review of the literature. Int. J. Mol. Sci. 17, E688 (2016).

23. Peltola, H., Rød, T.O., Jónsdóttir, K., Böttiger, M. \& Coolidge, J.A. Life-threatening Haemophilus influenzae infections in Scandinavia: a five-country analysis of the incidence and the main clinical and bacteriologic characteristics. Rev. Infect. Dis. 12, 708-715 (1990).

24. Tristram, S., Jacobs, M.R. \& Appelbaum, P.C. Antimicrobial resistance in Haemophilus influenzae. Clin. Microbiol. Rev. 20, 368-389 (2007).

25. Hoban, D. \& Felmingham, D. The PROTEKT surveillance study: antimicrobial susceptibility of Haemophilus influenzae and Moraxella catarrhalis from community-acquired respiratory tract infections. J. Antimicrob. Chemother. 50 (Suppl. S1), 49-59 (2002)

26. Peltola, $\mathrm{H}$. et al. Perspective: a five-country analysis of the impact of four different Haemophilus influenzae type b conjugates and vaccination strategies in Scandinavia. J. Infect. Dis. 179, 223-229 (1999).

27. Adam, H.J. et al. Changing epidemiology of invasive Haemophilus influenzae in Ontario, Canada: evidence for herd effects and strain replacement due to Hib vaccination. Vaccine 28, 4073-4078 (2010).

28. Public Health Agency of Canada. Vaccine preventable disease: surveillance report to December 31, 2015 (Public Health Agency of Canada, Ottawa, 2017).

29. Hargreaves, R.M., Slack, M.P., Howard, A.J., Anderson, E. \& Ramsay, M.E. Changing patterns of invasive Haemophilus influenzae disease in England and Wales after introduction of the Hib vaccination programme. Br. Med. J. 312, 160-161 (1996).

30. Heilmann, K.P. et al. Decreasing prevalence of $\beta$-lactamase production among respiratory tract isolates of Haemophilus influenzae in the United States. Antimicrob. Agents Chemother. 49, 2561-2564 (2005).

31. Hasegawa, K. et al. Diversity of ampicillin-resistance genes in Haemophilus influenzae in Japan and the United States. Microb. Drug Resist. 9, 39-46 (2003).

32. Feldman, C. \& Anderson, R. Epidemiology, virulence factors and management of the pneumococcus. F1000Res, 5, 2320 (2016).

33. Tomczyk, S. et al. Prevention of antibiotic-nonsusceptible invasive pneumococcal disease with the 13-valent pneumococcal conjugate vaccine. Clin. Infect. Dis. 62 , 1119-1125 (2016).

34. Hampton, L.M. et al. Prevention of antibiotic-nonsusceptible Streptococcus pneumoniae with conjugate vaccines. J. Infect. Dis. 205, 401-411 (2012).

35. Cohen, R., Cohen, J.F., Chalumeau, M. \& Levy, C. Impact of pneumococcal conjugate vaccines for children in high- and non-high-income countries. Expert Rev. Vaccines 16, 625-640 (2017).

36. Moore, M.R. et al. Effectiveness of 13-valent pneumococcal conjugate vaccine for prevention of invasive pneumococcal disease in children in the USA: a matched casecontrol study. Lancet Respir. Med. 4, 399-406 (2016).

37. Knol, M.J. et al. Invasive pneumococcal disease 3 years after introduction of 10 -valent pneumococcal conjugate vaccine, the Netherlands. Emerg. Infect. Dis. 21, 2040-2044 (2015).

38. Kash, J.C. \& Taubenberger, J.K. The role of viral, host, and secondary bacterial factors in influenza pathogenesis. Am. J. Pathol. 185, 1528-1536 (2015).

39. Ozgur, S.K. et al. Effectiveness of inactivated influenza vaccine for prevention of otitis media in children. Pediatr. Infect. Dis. J. 25, 401-404 (2006).

40. Glezen, W.P., Taber, L.H., Frank, A.L. \& Kasel, J.A. Risk of primary infection and reinfection with respiratory syncytial virus. Am. J. Dis. Child. 140, 543-546 (1986).

41. Nair, H. et al. Global burden of acute lower respiratory infections due to respiratory syncytial virus in young children: a systematic review and meta-analysis. Lancet $\mathbf{3 7 5}$ 1545-1555 (2010)

42. American Academy of Pediatrics, Committee on Infectious Diseases and Bronchiolitis Guidelines Committee. Updated guidance for palivizumab prophylaxis among infants and young children at increased risk of hospitalization for respiratory syncytial virus infection. Pediatrics 134, 415-420 (2014).

43. Hament, J.-M., Kimpen, J.L.L., Fleer, A. \& Wolfs, T.F.W. Respiratory viral infection predisposing for bacterial disease: a concise review. FEMS Immunol. Med. Microbiol. 26, 189-195 (1999).

44. Matias, G. et al. Estimates of mortality attributable to influenza and RSV in the United States during 1997-2009 by influenza type or subtype, age, cause of death, and risk status. Influenza Other Respir. Viruses 8, 507-515 (2014).

45. Falsey, A.R., Hennessey, P.A., Formica, M.A., Cox, C. \& Walsh, E.E. Respiratory syncytial virus infection in elderly and high-risk adults. N. Engl. J. Med. 352, 1749-1759 (2005).

46. Widmer, K. et al. Rates of hospitalizations for respiratory syncytial virus, human metapneumovirus, and influenza virus in older adults. J. Infect. Dis. 206, 56-62 (2012). 
47. Zhou, H. et al. Hospitalizations associated with influenza and respiratory syncytial virus in the United States, 1993-2008. Clin. Infect. Dis. 54, 1427-1436 (2012).

48. Rey-Jurado, E. \& Kalergis, A.M. Immunological features of respiratory syncytial viruscaused pneumonia-implications for vaccine design. Int. J. Mol. Sci. 18, E556 (2017).

49. Openshaw, P.J.M., Chiu, C., Culley, F.J. \& Johansson, C. Protective and harmful immunity to RSV infection. Annu. Rev. Immunol. 35, 501-532 (2017).

50. Walsh, E.E. Respiratory syncytial virus infection: an illness for all ages. Clin. Chest Med. 38, 29-36 (2017).

51. Beadling, C. \& Slifka, M.K. How do viral infections predispose patients to bacterial infections? Curr. Opin. Infect. Dis. 17, 185-191 (2004).

52. Simoes, E.A., Tan, D.H., Ohlsson, A., Sales, V. \& Wang, E.E. Respiratory syncytial virus vaccine: a systematic overview with emphasis on respiratory syncytial virus subunit vaccines. Vaccine 20, 954-960 (2001).

53. Falsey, A.R. et al. Comparison of the safety and immunogenicity of 2 respiratory syncytial virus (RSV) vaccines - nonadjuvanted vaccine or vaccine adjuvanted with alumgiven concomitantly with influenza vaccine to high-risk elderly individuals. J. Infect. Dis. 198, 1317-1326 (2008).

54. Jenkins, P.J. et al. Invasive group B streptococcal disease in an orthopaedic unit. J. Hosp. Infect. 76, 231-233 (2010).

55. Ngwuta, J.O. et al. Prefusion F-specific antibodies determine the magnitude of RSV neutralizing activity in human sera. Science Transl. Med. 7, 309ra162 (2015).

56. Cohen, S.H. et al. Clinical practice guidelines for Clostridium difficile infection in adults: 2010 update by the Society for Healthcare Epidemiology of America (SHEA) and the Infectious Diseases Society of America (IDSA). Infect. Control Hosp. Epidemiol. 31, 431-455 (2010).

57. Wilcox, M.H. et al. Bezlotoxumab for prevention of recurrent Clostridium difficile infection. N. Engl. J. Med. 376, 305-317 (2017).

58. Sheldon, E. et al. A phase 1, placebo-controlled, randomized study of the safety, tolerability, and immunogenicity of a Clostridium difficile vaccine administered with or without aluminum hydroxide in healthy adults. Vaccine 34, 2082-2091 (2016).

59. Aguinagalde, L. et al. Emergence of amoxicillin-resistant variants of Spain9V-ST156 pneumococci expressing serotype $11 \mathrm{~A}$ correlates with their ability to evade the host immune response. PLoS One 10, e0137565 (2015).

60 . Yildirim, I. et al. Serotype specific invasive capacity and persistent reduction in invasive pneumococcal disease. Vaccine 29, 283-288 (2010).

61. Salkind, A.R. \& Rao, K.C. Antiobiotic prophylaxis to prevent surgical site infections. Am. Fam. Physician 83, 585-590 (2011).

62. Awad, S.S. Adherence to surgical care improvement project measures and post-operative surgical site infections. Surg. Infect. (Larchmt) 13, 234-237 (2012).

63. Mohamed, N. et al. Vaccine development to prevent Staphylococcus aureus surgicalsite infections. Br. J. Surg. 104, e41-e54 (2017).

64. Dayan, G.H. et al. Staphylococcus aureus: the current state of disease, pathophysiology and strategies for prevention. Expert Rev. Vaccines 15, 1373-1392 (2016).

65. Hiramatsu, K. et al. Methicillin-resistant Staphylococcus aureus clinical strain with reduced vancomycin susceptibility. J. Antimicrob. Chemother. 40, 135-136 (1997).

66. Hiramatsu, K. et al. Dissemination in Japanese hospitals of strains of Staphylococcus aureus heterogeneously resistant to vancomycin. Lancet 350, 1670-1673 (1997).

67. Caroom, C., Tullar, J.M., Benton, E.G.J. Jr., Jones, J.R. \& Chaput, C.D. Intrawound vancomycin powder reduces surgical site infections in posterior cervical fusion. Spine 38, 1183-1187 (2013)

68. Gaviola, M.L., McMillian, W.D., Ames, S.E., Endicott, J.A. \& Alston, W.K. A retrospective study on the protective effects of topical vancomycin in patients undergoing multilevel spinal fusion. Pharmacotherapy 36, 19-25 (2016).

69. Middleton, J.R. Staphylococcus aureus antigens and challenges in vaccine development. Expert Rev. Vaccines 7, 805-815 (2008).

70. Schaffer, A.C. \& Lee, J.C. Staphylococcal vaccines and immunotherapies. Infect. Dis. Clin. North Am. 23, 153-171 (2009).

71. Simioni, J. et al. A comparison of intestinal microbiota in a population of low-risk infants exposed and not exposed to intrapartum antibiotics: The Baby \& Microbiota of the Intestine cohort study protocol. BMC Pediatr. 16, 183 (2016).

72. Arboleya, S. et al. Impact of prematurity and perinatal antibiotics on the developing intestinal microbiota: a functional inference study. Int. J. Mol. Sci. 17, 649 (2016).

73. World Health Organization. Global Priority List of Antibiotic-Resistant Bacteria to Guide
Research, Discovery, and Development of New Antibiotics (World Health Organizaiton, 2017)

74. Walsh, T.R., Weeks, J., Livermore, D.M. \& Toleman, M.A. Dissemination of NDM-1 positive bacteria in the New Delhi environment and its implications for human health an environmental point prevalence study. Lancet Infect. Dis. 11, 355-362 (2011).

75. Yong, D. et al. Characterization of a new metallo- $\beta$-lactamase gene, bla $a_{\mathrm{NDM}-1}$, and a novel erythromycin esterase gene carried on a unique genetic structure in Klebsiella pneumoniae sequence type 14 from India. Antimicrob. Agents Chemother. 53, 5046 5054 (2009).

76. McGann, P. et al. Escherichia coli harboring $m c r-1$ and bla $a_{\text {CTX-M }}$ on a novel IncF plasmid: first report of $m c r-1$ in the United States. Antimicrob. Agents Chemother. 60, 4420-4421 (2016).

77. Poolman, J.T. \& Wacker, M. Extraintestinal pathogenic Escherichia coli, a common human pathogen: challenges for vaccine development and progress in the field. J. Infect. Dis. 213, 6-13 (2016).

78. Huttner, A. et al. Safety, immunogenicity, and preliminary clinical efficacy of a vaccine against extraintestinal pathogenic Escherichia coli in women with a history of recurrent urinary tract infection: a randomised, single-blind, placebo-controlled phase $1 \mathrm{~b}$ trial. Lancet Infect. Dis. 17, 528-537 (2017)

79. Lundberg, U., Senn, B.M., Schüler, W., Meinke, A. \& Hanner, M. Identification and characterization of antigens as vaccine candidates against Klebsiella pneumoniae. Hum. Vaccin. Immunother. 9, 497-505 (2013).

80. Mike, L.A., Smith, S.N., Sumner, C.A., Eaton, K.A \& Mobley, H.L. Siderophore vaccine conjugates protect against uropathogenic Escherichia coli urinary tract infection. Proc. Natl. Acad. Sci. USA 113, 13468-13473 (2016)

81. Jerse, A.E., Bash, M.C. \& Russell, M.W. Vaccines against gonorrhea: current status and future challenges. Vaccine 32, 1579-1587 (2014).

82. World Health Organization. Global Tuberculosis Report (World Health Organization 2017).

83. Kaufmann, S.H. et al. Progress in tuberculosis vaccine development and host-directed therapies-a state of the art review. Lancet Respir. Med. 2, 301-320 (2014).

84. von Reyn, C.F. et al. Prevention of tuberculosis in Bacille Calmette-Guérin-primed, HIV-infected adults boosted with an inactivated whole-cell mycobacterial vaccine. AIDS 24, 675-685 (2010)

85. World Health Organization. Global tuberculosis report (WHO Press, 2016).

86. de Bruyn, G. et al. Defining the optimal formulation and schedule of a candidate toxoid vaccine against Clostridium difficile infection: a randomized phase 2 clinical trial. Vaccine 34, 2170-2178 (2016).

87. Bézay, N. et al. Safety, immunogenicity and dose response of VLA84, a new vaccine candidate against Clostridium difficile, in healthy volunteers. Vaccine 34, 2585-2592 (2016).

88. Frenck, R.W. Jr. et al. Safety, tolerability, and immunogenicity of a 4-antigen Staphylococcus aureus vaccine (SA4Ag): results from a first-in-human randomised, placebo-controlled phase 1/2 study. Vaccine 35, 375-384 (2017).

89. Mancini, F. et al. One dose of Staphylococcus aureus 4C-Staph vaccine formulated with a novel TLR7-dependent adjuvant rapidly protects mice through antibodies, effector CD4+ T cells, and IL-17A. PLoS One 11, e0147767 (2016).

90. Leroux-Roels, G. et al. A randomized, observer-blind phase Ib study to identify formulations and vaccine schedules of a trivalent group B Streptococcus vaccine for use in non-pregnant and pregnant women. Vaccine 34, 1786-1791 (2016).

91. Stålhammar-Carlemalm, M. et al. Nonimmunodominant regions are effective as building blocks in a streptococcal fusion protein vaccine. Cell Host Microbe 2, 427-434 (2007).

92. Huttner, A. et al. Safety, immunogenicity, and preliminary clinical efficacy of a vaccine against extraintestinal pathogenic Escherichia coli in women with a history of recurrent urinary tract infection: a randomised, single-blind, placebo-controlled phase $1 \mathrm{~b}$ trial. Lancet Infect. Dis. 17, 528-537 (2017)

93. Langermann, S. et al. Vaccination with FimH adhesin protects cynomolgus monkeys from colonization and infection by uropathogenic Escherichia coli. J. Infect. Dis. 181, 774-778 (2000).

94. van den Dobbelsteen, G.P.J.M. et al. Immunogenicity and safety of a tetravalent E. coli O-antigen bioconjugate vaccine in animal models. Vaccine 34, 4152-4160 (2016).

95. Cawein, A. et al. Human capital gaps in vaccine development: an issue for global vaccine development and global health. Ann. N. Y. Acad. Sci. 1395, 3-11 (2017). 


\section{REVIEW}

COMPETING FINANCIAL INTERESTS

All authors are employees of Pfizer, Inc. 